\title{
Characterization of Vegetative Incompatibility in Morchella importuna and Location of the Related-Genes by Bulk Segregant Analysis
}

\author{
Hongmei Chai ${ }^{1,2,3+}$, Ping Liu ${ }^{1,2,3 \dagger}$, Yuanhao Ma ${ }^{1,2,3}$, Weimin Chen ${ }^{1,2,3}$, Nan Tao ${ }^{1,2,3}$ and \\ Yongchang Zhao ${ }^{1,2,3 *}$
}

'Biotechnology and Germplasmic Resource Research Institute, Yunnan Academy of Agricultural Sciences, Kunming, China, ${ }^{2}$ Yunnan Provincial Key Laboratory of Agricultural Biotechnology, Kunming, China, ${ }^{3}$ Key Laboratory of Southwestern Crop Resource Germplasm Innovation, Ministry of Agriculture and Rural Affairs, Kunming, China

OPEN ACCESS

Edited by:

James Hane,

Curtin University, Australia

Reviewed by:

Robin Ohm

Utrecht University, Netherlands

Jaehyuk Choi,

Incheon National University,

South Korea

*Correspondence:

Yongchang Zhao

yaasmushroom@aliyun.com

${ }^{\dagger}$ These authors have contributed equally to this work

Specialty section:

This article was submitted to Evolutionary and Genomic

Microbiology,

a section of the journa

Frontiers in Microbiology

Received: 03 December 2021 Accepted: 31 January 2022

Published: 07 March 2022

Citation:

Chai H, Liu P, Ma Y, Chen W,

Tao $N$ and Zhao $Y$ (2022)

Characterization of Vegetative Incompatibility in Morchella importuna and Location of the Related-Genes by

Bulk Segregant Analysis.

Front. Microbiol. 13:828514. doi: 10.3389/fmicb.2022.828514
Vegetative incompatibility $(\mathrm{VI})$ is a widespread phenomenon developed in Morchella importuna, a species of ascomycete fungus that is cultivated on a rapidly expanding scale in China. Understanding the genetic bases of this nonself-recognition phenomenon is beneficial for resolving some problems that are associated with the production of this highly prized edible fungus, such as crossbreeding, strain classification, and pathogen transmission. VI is genetically controlled by het genes, organized in two different systems, namely allelic and nonallelic. These het genes have been well characterized in Podospora anserina and Neurospora crassa. In this work, putative het-homologs were identified in the genome of $M$. importuna, but their low allelic polymorphism in different vegetative compatibility groups (VCGs) suggested that $\mathrm{VI}$ in this fungus might not be regulated by these het genes. The progeny derived from vegetative compatible parents became a VCG, while the single-ascospore strains from vegetative incompatible parents were divided into four VCGs, and the interaction between the inter-group strains led to the formation of two types of barrages, viz., thin dark line and raised aggregate of hyphae. The Bulk Segregant Analysis confirmed that the genes mimpvic32 and mimpvic33 were linked to VI reactions in $\mathrm{M}$. importuna; nevertheless, the formation of barrages also occurred between the pairs carrying the same allele of these two genes. In sum, the VI control system in M. importuna was complicated, and there were more other allelic or non-allelic VI-related genes.

Keywords: allelic polymorphism, ascomycete, barrage, het gene, vegetative compatibility group

\section{INTRODUCTION}

In filamentous ascomycete fungi, the self/nonself-recognition of conspecific individuals occurs during sexual and asexual phases in the life cycle. At the sexual stage, the recognition is controlled by a single mating-type locus with two idiomorphs termed MAT1-1 and MAT1-2 (Turgeon and Yoder, 2000), and individuals of heterothallic species that carry the opposite mating types are sexually 
compatible and thus could mate to produce offspring (Debuchy and Turgeon, 2006). During the asexual phase, vegetative incompatibility (VI) systems are responsible for recognition and limit the fusion of genetically distinct mycelia. Some forms of demarcation referred to as barrages, including pigmentation, shear belt, and hyphal aggregates, may occur in the zone of contact where two incompatible individuals meet (Cristina and Myron, 2003; Esser, 2016). The molecular genetic basis of VI has been studied in detail in the ascomycete species Podospora anserina and Neurospora crassa (Paoletti, 2016). The VI is controlled by multiple, unlinked het loci (Glass and Kuldau, 1992), which act as allelic and nonallelic systems. In P. anserina, incompatibility is caused by the coexpression of het-s/het-S of the same locus (allelic systems) and controlled by nonallelic systems, including het-c/het-d, het-r/het- $v$, and het-c/het-e (Saupe et al., 2001). For $N$. crassa, in addition to nonallelic pinc/het-c, and het-6/un-24, the allelic mating-type idiomorph is involved in incompatibility reaction; however, the expression of a gene named tol has been postulated to allow the coexistence of opposite mating-type nuclei during the sexual reproductive phase (Daskalov et al., 2017). The majority of these het genes characterize a protein containing a HET domain (Pfam06985), with approximately 150 amino acids (Espagne et al., 2002; Zhao et al., 2015), which plays a fundamental role as the mediator of VI-associated programmed cell death (PCD).

Nonetheless, research indicates that self and nonselfrecognition may not be regulated by the het loci in some cases or certain species. Micali and Smith (2003) found that in N. crassa, barrages were observed to occur between strains that were identical at all major het loci, as well as the cases where barrages did not form between strains that had genetic differences at het-6, het-c, and/or mat. In the genome of Tuber melanosporum, variable numbers of homologous sequences of VI-like genes were detected, but the expression data of these genes, along with low allelic polymorphism, suggested that they might not mediate VI in T. melanosporum (Iotti et al., 2012).

Morchella importuna is a great edible fungus belonging to the Ascomycota, Discomycetes, Pezizales, and Morchellaceae. In recent years, the successful field cultivation of $M$. importuna has been achieved in China and gradually expanded (Liu et al., 2017). Therefore, it is important to develop suitable strains by crossbreeding of parents with particular characteristics; meanwhile, the corresponding genetic basis should be studied indepth. The sexual reproduction of $M$. importuna, a heterothallic organism, requires two haploid strains containing MAT1-1 and MAT1-2 idiomorphs (Chai et al., 2017). However, sexually compatible strains often exhibit VI, and there was no correlation between the mating type and vegetative compatibility (Liu et al., 2021). Moreover, VI was ubiquitous among the M. importuna strains (Ma et al., 2017; Liu et al., 2021). To date, no het genes have been identified in Morchella spp.

The objectives of this study were first to describe the barrage types in $M$. importuna and second to determine whether the reported het genes regulate VI, and finally to further identify VI-regulated genes through the Bulk Segregant Analysis (BSA) using single-ascospore populations from vegetatively incompatible parents.

\section{MATERIALS AND METHODS}

\section{Strains and Isolation of Single Ascospores}

Strains YAASMYPL6-3 and YAASMYPL6-1, verified as MAT11 and MAT1-2 mating-types, respectively (Chai et al., 2017), as well as vegetatively incompatible, were isolated from an ascocarp YAASMYPL6 of $M$. importuna. The crossbreeding of YAASMYPL6-3 and YAASMYPL6-1 was performed by mixing equal parts of the final spawn, followed by the cultivation in a farm in Jinning District (Kunming City, Yunnan Province, China). Among the offspring of the harvest, three ascocarps, namely Y1Y3-1, Y1Y3-2, and Y1Y3-3, with matured ascospores, were selected to develop the single-ascospore populations.

The wild ascocarp Zhao0001 of M. importuna was collected from the Yulong County (Yunnan Province, China), and the ascospore print was provided by Dr. Zhao Qi (Kunming Institute of Botany, Academia Sinica). The ascocarps 6611-5 and 6611-7 were obtained from the fields of morel cultivation in the Wuding County (Yunnan Province, China), and their parent strain 6611 was also provided by Dr. Zhao.

For the isolation of single ascospores, a pool of spores with proper initial concentrations was prepared, as reported by Liu et al. (2021). To rule out two or more spores isolated together, each ascospore was collected under a microscope using a small glass capillary tube and then transferred to a $5-\mathrm{cm}$ Petri dish containing potato dextrose agar (PDA). After maintaining $24 \mathrm{~h}$ in dark at $22^{\circ} \mathrm{C}$, the Petri dish was taken for examination under a dissecting microscope, and the germinated spore colony was transferred to a new Petri dish. Every single-ascospore isolate was numbered with Arabic numerals corresponding to the ascocarp (Table 1) and stored in the Mushroom Center of Yunnan Germplasm Bank of Crops in Yunnan Academy of Agricultural Sciences, Kunming, China.

\section{Strain Confrontations}

It would be a huge numbers of combinations if one-toone pairing were performed in the isolates obtained from the ascocarps Y1Y3-1, Y1Y3-2, and Y1Y3-3. Therefore, firstly, confrontational culture was carried out between every singleascospore strain and the parent strains YAASMYPL6-3 and YAASMYPL6-1, and then the strains were divided into groups based on vegetative compatibility reactions. Secondly, three

TABLE 1 | Single-ascospore populations used in this study.

\begin{tabular}{lcc}
\hline $\begin{array}{l}\text { Single-ascospore } \\
\text { populations }\end{array}$ & Ascocarp & Ascocarp characteristic \\
\hline Y1Y3-001-Y1Y3-200 & Y1Y3-1 & $\begin{array}{r}\text { Progeny from YAASMYPL6-3 and } \\
\text { YAASMYPL6-1 crossed }\end{array}$ \\
Y1Y3-201-Y1Y3-400 & Y1Y3-2 & Wild \\
Y1Y3-401-Y1Y3-415 & Y1Y3-3 & Cultivation \\
Zhao0001-1- & Zhao0001 & \\
Zhao0001-33 & & \\
$6611-5-1-6611-5-13$ & $6611-5$ & $6611-7$
\end{tabular}


isolates were randomly chosen from each group to test their confrontational interactions with members of the intra-group and the inter-group, and therefore the vegetative compatibility groups (VCGs) were verified.

According to Chen and Zhang (2010), seven isolates could be paired in all possible combinations in three Petri dishes. For the single-ascospore isolates derived from the ascocarps Zhao0001, 6611-5, and 6611-7, vegetative compatibility assays were performed within the population, and each test was repeated three times in a $9-\mathrm{cm}$ Petri dish.

\section{Molecular Biology Assays}

Total DNA was isolated from the 7-day-old mycelia grown in PDA using the Fungal gDNA Kit (GD2416) (Biomiga, San Diego, CA, United States). Polymerase chain reaction (PCR) was performed in a $25-\mu \mathrm{L}$ mixture containing $12.5 \mu \mathrm{L}$ of PCR Mix (Vazyme Biotech Co., Ltd, Nanjing, China), $1 \mu \mathrm{L}$ of each $10 \mu \mathrm{M}$ primer, and $1 \mu \mathrm{L}$ of $10 \mathrm{ng} / \mu \mathrm{L}$ genomic DNA.

Primer pairs for PCR were designed using the software Primer Premier 5.0 (Premier Biosoft International, Palo Alto, CA, United States), and their detailed information, along with amplification conditions, are listed in Supplementary Table 1. The synthesis of primer pairs and sequencing of all PCR products were carried out in Beijing Tsingke Biotechnology. As reported by Chai et al. (2017), the method was used to analyze the mating types of single-ascospore strains.

\section{Analysis of het-Homologs in Morchella importuna}

Genome databases of $M$. importuna, including YAASMYPL6-3 (GWHBCHL00000000) and YAASMYPL61 (GWHBCHM00000000), have been deposited in China National Center for Bioinformation. The VI-related genes in $N$. crassa, P. anserina, and T. melanosporum were selected according to a study conducted by Mirco et al. (2012) and are listed in Table 2. Searches were run against the genome database of YAASMYPL6-3 using tBLASTn algorithms (Altschul et al., 1997 ). Genes with an $E$-value of $<\mathrm{e}^{-10}$ and $>30 \%$ identity were selected as targets.

Primer pairs, shown in Supplementary Table 1, were designed to amplify the corresponding fragment in some members of each VCG. Amplified products were prepared for sequencing, and sequence polymorphism analyses were performed using Clustalx 1.83 (Jeannmougin et al., 1998). Sequences of het-homologs in some $M$. importuna strains were submitted to GenBank under the accession numbers OL303938-OL303979.

\section{The Bulk Segregant Analysis}

Based on the VI reactions with the parent strains YAASMYPL63 and YAASMYPL6-1, the progenies were divided into two VCGs. Sixty strains were selected randomly from each VCG to isolate the genomic DNA, and then DNA samples were pooled by combining equal amounts from each sample to form two DNA bulks. Genomic libraries from two VCG pools and two parents were constructed, and sequencing was performed using the Illumina $\mathrm{HiSeq}^{\mathrm{TM}} 2000$ platform by
Beijing Biomarker Technologies, and corresponding metadata files were submitted to GenBank under the accession numbers SRR17634835 SRR17634835. The clean reads of each VCG were aligned to the reference genome YAASMYPL6-3 using BurrowsWheeler Aligner (BWA v0.7.10) (Li and Durbin, 2009), and multiple read pairs with external coordinates were removed using the Mark Duplicate software of Picard. ${ }^{1}$ Local Realignment, Base Recalibration, Single Nucleotide Polymorphism (SNP), and Insertion-Deletion (InDel) calling were performed using the GATK (McKenna et al., 2010) software. The SnpEff software (Cingolani et al., 2012) was used to annotate SNPs and small InDels based on the GFF files of the reference genome.

The SNPs and small InDels (1-5 bp), supported by fewer than four reads, with multiple genotypes but no polymorphism between the two VCG bulks, were eliminated. After filtering, the ED (Altschul et al., 1997) and SNP-index and InDel-index algorithms (Deng et al., 2006; Ashburner et al., 2010) were performed to obtain significant genetic differences between bulks. The higher the ED value and the closer to 1 the $\Delta$ (SNPindex) and $\Delta$ (InDel-index) values, the closer the distance to the targeted sites. The ED median $+3 \mathrm{SD}$ of the fitted value at all loci was set as the association threshold. The numbers of association SNPs and small InDels in every scaffold were counted, and the scaffolds enriched with candidate sites were screened by setting the threshold of FDR $<0.01$. Finally, the candidate scaffolds were obtained by combining ED algorithms and SNP-index algorithms.

\section{RESULTS}

\section{Homologs of het Genes in the Genome of Morchella importuna}

The tBLASTn searches were run against the YAASMYPL6-3 genome using an $E$-value of $<\mathrm{e}^{-10}$ and $>30 \%$ identity as the filter threshold, and variable numbers of homologous sequences were identified only for het-c, un-24, het-c2, temelhet 45 , and temelhet21. No homologues were detected for the pin-c, het-6, tol, het-e (het-d/het-r), and het-s (hetS) (Table 2).

In the genome YAASMYPL6-3, three predicted genes, namely GME5750, GME1887, and GME3821, were simultaneously identified for each het-c gene in N. crassa, and all contained a conserved HET-C domain (PF07217). The detected homolog GME2544 showed more than $70 \%$ identity to $u n-24^{\circ R}$ and $u n$ $24^{P A}$, but the high sequence similarity was only limited to the residues of the first $\mathrm{N}$-terminal ribonucleoside reductase domain (PF02867), with more difference at their C-terminal.

The tBLASTn searches were run using the VI-related genes in $P$. anserina as the query, and only the homolog GME2473 for het-c2 was identified, which contained the glycolipid transfer protein domain (PF08718). For het-e, het-d, and het- $r$, more than 50 predicted genes with an $E$-value of $<\mathrm{e}^{-10}$ were retrieved, many of which only displayed low similarity at the C-terminal WDrepeat domain, but a few of them showed low identity to the central NACHT (PF05729), and none was characterized by the

\footnotetext{
${ }^{1}$ http://sourceforge.net/projects/picard/
} 
TABLE 2 | The het-homologs in the YAASMYPL6-3 genome of M. importuna.

\begin{tabular}{|c|c|c|c|c|c|c|}
\hline Species & het & GenBank number & Het-homologues in YAASMYPL6-3 & Scaffold in YAASMYPL6-3 & Identities (\%) & $E$-value \\
\hline \multirow[t]{16}{*}{ N. crassa } & het- $c^{\circ R}$ & AAB48349 & GME5750 & Scaffold 380 & 57 & 0 \\
\hline & & & GME1887 & Scaffold 77 & 43 & $3 e^{-151}$ \\
\hline & & & GME3821 & Scaffold 200 & 44 & $4 e^{-146}$ \\
\hline & het-c $c^{P A}$ & AAF08294 & GME5750 & Scaffold 380 & 57 & 0 \\
\hline & & & GME1887 & Scaffold 77 & 42 & $1 e^{-149}$ \\
\hline & & & GME3821 & Scaffold 200 & 44 & $4 e^{-148}$ \\
\hline & het-c ${ }^{G R}$ & AAF08295 & GME5750 & Scaffold 380 & 56 & 0 \\
\hline & & & GME1887 & Scaffold 77 & 47 & $1 e^{-149}$ \\
\hline & & & GME3821 & Scaffold 200 & 44 & $5 e^{-146}$ \\
\hline & pin-c1 & ABC46540 & - & & & \\
\hline & Pin-c2 & ABC46541 & - & & & \\
\hline & pin-c3 & ABC46542 & - & & & \\
\hline & het- $6^{\circ R}$ & Q9UV10 & - & & & \\
\hline & $u n-24^{\circ} R$ & Q9UW15 & GME2544 & Scaffold 116 & 71 & 0 \\
\hline & $u n-24^{P A}$ & ABF71875 & GME2544 & Scaffold 116 & 72 & 0 \\
\hline & tol & AAC64945 & - & & & \\
\hline \multirow[t]{6}{*}{ P. anserina } & het-c2 & AAA20542 & GME2473 & Scaffold 113 & 50 & $1 e^{-58}$ \\
\hline & het-e & CAL30215 & - & & & \\
\hline & het-d & CAL30216 & - & & & \\
\hline & het-r & ACM48730 & - & & & \\
\hline & het-s & 1718317A & - & & & \\
\hline & het-S & 1718317B & - & & & \\
\hline \multirow[t]{3}{*}{ T. melanosporum } & tmelhet45 & AFB74445 & GME5583 & Scaffold 362 & 35 & $2 e^{-34}$ \\
\hline & & & GEM5612 & Scaffold 366 & 30 & $4 e^{-31}$ \\
\hline & tmelhet21 & AFB74447 & - & & & \\
\hline
\end{tabular}

presence of the N-terminal HET domain. Thus, these retrieved genes were not considered as the candidates.

Two predicted genes GME5583 and GME5612 were detected when the tBLASTn search was performed using the VI-related protein TMELHET45 in T. melanosporum as a query. To further investigate the HET-domain-containing genes in the YAASMYPL6-3 genome, tBLASTn searches were also conducted using only HET domain sequences of all selected het genes in N. crassa, P. anserina, and T. melanosporum, but no more homologs were identified.

\section{Mycelial Interactions in Morchella importuna}

In this study, almost 10,000 confrontation tests were performed with 100 strains of $M$. importuna. According to the phenotypic features of the antagonistic reaction, barrages could be divided into type I and type II.

For type I, a dark reddish-brown line formed a contact zone, and the width was about $0.5-2 \mathrm{~mm}$. After inoculation for 34 days, the hyphal branches grew close to each other, and an obvious border-zone infarct could be observed (Figure 1A). At 7-10 days, the pigment was formed in the hyphae, and the border zone turned to an evident dark line, but the width of the zone did not show an obvious change (Figures 1B,C). Except for the dark line formed, the confronting strains grew normally.
In some pairs, the type II barrage occurred, whereas in three cases, mycelia were aggregated to form a dense hyphal mat. First, the dense hyphae could extend out along the interaction zone and develop to barrages ranging from 0.5 to $2 \mathrm{~cm}$ (Figure 1D), and a wide, deep yellow-brown border could be observed from the bottom of the Petri dish (Figure 1E). In another case, the dense hyphae formed two prominent fan-shaped mats on one side of the pairs (Figure 1F). Finally, hyphal aggregates appeared on one side of the pairs, and another confronting strain grew normally (Figure 1G). The above-mentioned cases could occur simultaneously with three replications for the same pairs (Figures 1H-J), and thus the three antagonistic phenomena were classified as the type II barrage.

Among the strains with vegetative compatibility, lines of demarcation were not observed, and the hyphae from paired interactions grew close to each other when strains were confronted (Figures 1K,L). But sometimes, sclerotia might be concentrated on one side of the confronting strain (Figure 1M), or along the contact zone (Figure 1N), or at the angle between the contact zone and the Petri dish (Figure 10). These phenomena randomly occurred and were not specific to certain strains.

\section{The Determination of Vegetative Compatibility Groups}

A total of 415 single-ascospore strains were isolated from the ascocarps Y1Y3-1, Y1Y3-2, and Y1Y3-3. By confrontation assays 


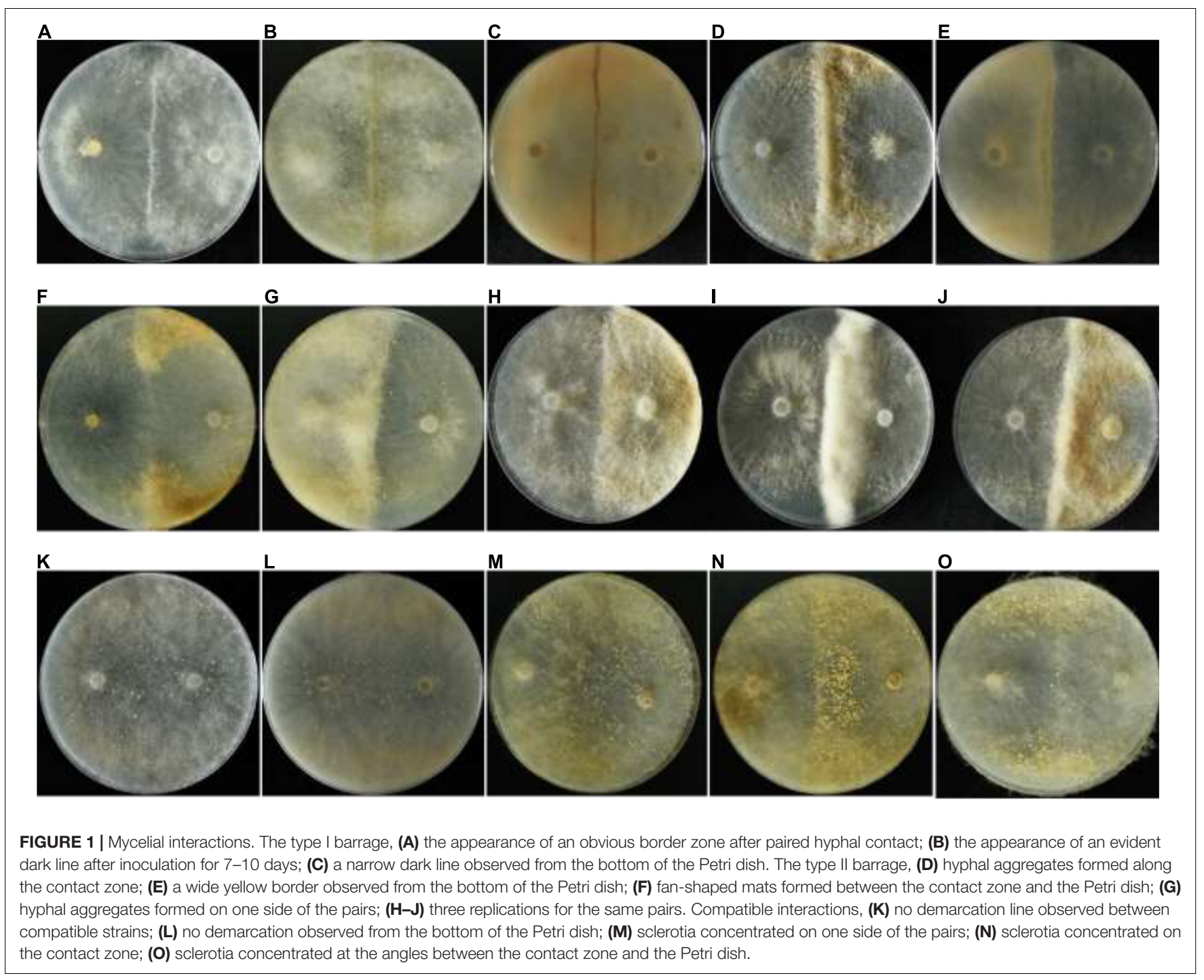

with the parent strains YAASMYPL6-3 and YAASMYPL6-1, the progenies were divided into four VCGs. A total of 118 strains showed the type I barrage with YAASMYPL6-3, while with YAASMYPL6-1, they compatibly grew and were classified as VCGA1 (Figure 2A). Ninety-nine strains exhibited type I barrage with YAASMYPL6-3 and type II barrage with YAASMYPL6-1, and were then categorized into VCGA2 (Figure 2B). Conversely, the type I barrage with YAASMYPL6-1 occurred for 96 VCGB1 strains, which had the vegetative compatibility with YAASMYPL6-3, whereas 83 identified VCGB2 strains showed type I barrage with YAASMYPL6-1 and type II barrage with YAASMYPL6-3. Due to slow growth, confrontation tests were not done with the other 19 strains.

Three strains were selected randomly from each VCG to carry out confrontation assays with intra-group and inter-group strains to assess vegetative compatibility relationships among the four groups. The results suggested that the type I barrage occurred between VCGA (VCGA1 and VCGA2) and VCGB (VCGB1 and VCGB2), while type II barrage was observed between VCGA1 and VCGA2 and between VCGB1 and VCGB2; meanwhile, the vegetative compatibility relationship among the members within a group was further confirmed. The results of confrontation tests between the selected 12 strains are presented in Table 3.

All $351[C(27,2)=(27 \times 26) / 2=351]$ combinations, made between the 27 strains 6611-5-1-6611-5-13, 6611-7-16611-7-13, and 6611, were analyzed. The results demonstrated that they were all vegetatively compatible and could be classified into a VCG, named VCG6611 (Figure 2C). All 630 [C(36, $2)=(36 \times 35) / 2=630]$ combinations of the confrontation tests with 36 strains Zhao0001-1-Zhao0001-33, YAASMYPL61, YAASMYPL6-3, and 6611 were made, and part of the results presented in Figure 2D. Except the combination of Zhao000128 and Zhao0001-29 was vegetative compatible, the other 629 combinations were vegetative incompatible and exhibited the type I barrage, as showed in Figure 2D between the strains Zhao0001-3 and Zhao0001-6, Zhao0001-8, Zhao0001-13, and Zhao0001-15. 


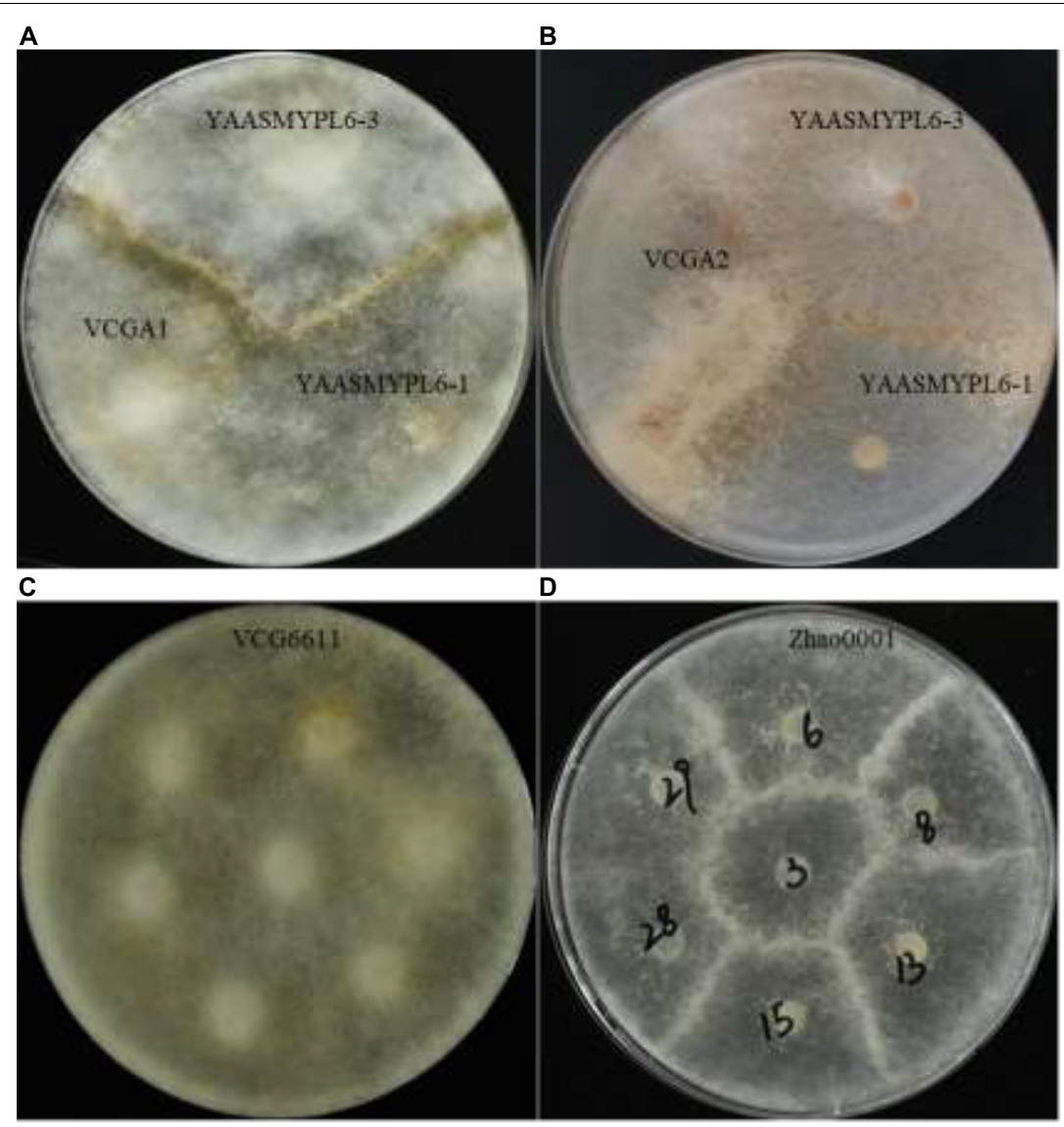

FIGURE 2 | The confrontation test against strains. (A) The interaction between the VCGA1 strain and the parent strains. (B) The interaction between the VCGA2 strain and the parent strains. (C) Interactions between seven strains from the VCG6611 population. (D) Interactions between seven strains from the Zhao0001 population; Zhao0001-28 and Zhao0001-29 strains were compatible.

Mating-type identifications of some strains randomly selected from each VCG were performed, and the results showed the presence of MAT1-1 and MAT1-2 strains in every VCG.

\section{Analysis of het-Homologs Polymorphisms}

Seven candidate sequences of het-homologs were derived from the genome YAASMYPL6-3, and the corresponding primer pairs, P24 to P30, were designed (Supplementary Table 1). DNA sequence polymorphism analysis was performed with 25 strains, including the members from VCGA1, VCGA2, VCGB1, VCGB2, VCG6611, as well as the Zhao0001 population (Supplementary Table 2).

The sequence alignment analysis of PCR products indicated that there were low levels of allelic polymorphism of these homologs among the members belonging to different VCGs. Amplified sequences of 25 strains were identical when the primer pairs P24, P25, P27, P28, and P29 were used. With the primer pair P26, four SNP loci were detected in 25 amplified PCR products; however, they were not distributed mainly in a particular VCG. In addition, two SNP loci were located in the introns, while the other two were non-synonymous coding. In the products amplified with the primer pair P30, two SNP loci were detected only in four Zhao0001 strains, and only one SNP locus was synonymous coding.

In conclusion, for each het-homolog, there were no allelic polymorphisms between the VCGs in this study, and they might not be able to regulate the VI in M. importuna.

\section{Location of Vegetative Incompatibility-Related Genes by Bulk Segregant Analysis}

Genomic libraries of VCGA and VCGB bulks and two parents were constructed, and sequencing was performed. A total of 15,752 SNPs and 4,140 InDels were detected by the alignment of each sample to the reference genome YAASMYPL6-3. After filtering, 7,627 SNPs and 1,753 InDels showed high quality for further association analysis. The Euclidean Distance (ED) algorithm was applied to measure allele segregation and to identify the linked scaffolds based on the SNPs between the two bulks. Using the median $+3 \mathrm{SD}$ of the fitted value at all loci as the cut-off, the association SNPs in every scaffold were identified, and eight scaffolds (FDR <0.01) which significantly enriched candidate SNPs were screened out (Supplementary Table 3). 
TABLE 3 | Results of the confrontation experiments between the 12 selected strains.

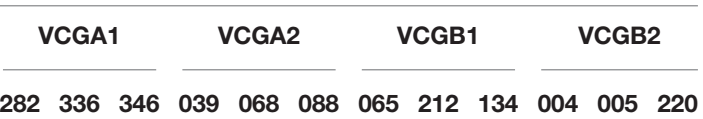

\begin{tabular}{|c|c|c|c|c|c|c|c|c|c|c|c|c|}
\hline \multirow[t]{3}{*}{ VCGA1 } & 282 & - & & & & & & & & & & \\
\hline & 336 & - & - & & & & & & & & & \\
\hline & 346 & - & - & - & & & & & & & & \\
\hline \multirow[t]{3}{*}{ VCGA2 } & 039 & $\|$ & $\|$ & II & - & & & & & & & \\
\hline & 068 & $\|$ & $\|$ & II & - & - & & & & & & \\
\hline & 088 & $\|$ & $\|$ & II & - & - & - & & & & & \\
\hline \multirow[t]{3}{*}{ VCGB1 } & 065 & 1 & I & 1 & 1 & 1 & I & - & & & & \\
\hline & 212 & I & 1 & 1 & 1 & 1 & 1 & - & - & & & \\
\hline & 134 & 1 & I & 1 & 1 & 1 & 1 & - & - & - & & \\
\hline \multirow[t]{3}{*}{ VCGB2 } & 004 & I & 1 & 1 & 1 & 1 & I & $\|$ & $\|$ & $\|$ & - & \\
\hline & 005 & 1 & 1 & 1 & 1 & 1 & 1 & $\|$ & $\|$ & $\|$ & - & - \\
\hline & 220 & I & I & I & I & I & I & $\|$ & $\|$ & $\|$ & - & - \\
\hline
\end{tabular}

The prefix "Y1Y3-" was omitted from all the strain numbers. -, vegetative compatibility; I, type I barrage; II, type I/ barrage.

Meanwhile, the SNP-index algorithm was performed to analyze significant differences in genotype frequencies between the two bulks, and the numbers of association SNPs in every scaffold were included in Supplementary Table 4 and 53 linked scaffolds (FDR <0.01) were identified. Eight overlapping scaffolds using these two algorithms represented the target scaffolds based on SNP analysis (Table 4). Similarly, small InDel association analysis was carried out using the ED and InDel-index algorithms, then 3 and 27 scaffolds (FDR $<0.01$ ) which enriched candidate sites were detected respectively (Supplementary Tables 5, 6). Three scaffolds could be found in the intersection of these two algorithms, which were the target scaffolds based on small InDel analysis (Table 4). Ultimately, these target scaffolds intersected to obtain the candidate scaffolds 50 and 751 (Table 4 and Figure 3A).

The analysis of the YAASMYPL6-1 and YAASMYPL6-3 genomes showed that the scaffolds 50 and 751 were closely connected, and they harbored 23 predicted genes (Figure 3B) of which annotation were listed in Supplementary Table 7. Among them, gene 4,12,16, and 17 were non-synonymous mutant genes between the two parents. Further analysis revealed that genes 12 and 16 harbored only one and two nonsynonymous SNPs respectively, however, gene 4 (mimpvic32) and 17 (mimpvic33) carried the vast majority of non-synonymous SNPs and InDels (Figure 3C), which were speculated to be VI-related candidate genes.

Sequences of mimpvic32 in the parental strain YAASMYPL6-1 were defined as A allele mimpvic32A, while B allele mimpvic32B was in the parental strain YAASMYPL6-3. The lengths of the mimpvic32A and mimpvic32B sequences were 2,906 and 2,875 bp, respectively, and both contained 8 introns and encoded proteins of 765 amino acids (Table 5). There was a sequence identity of 92.6\% between these amino acids, and sequence variances were mainly concentrated on C-terminal regions (Supplementary Figure 1). The BLASTx analysis revealed that the predicted protein contained a CorA-like $\mathrm{Mg}^{2+}$ transporter protein domain (PF01544), which is abundant in most bacteria and archaea, and in some eukaryotes (Kehres et al., 1998). The CorA proteins are generally involved in transporting $\mathrm{Mg}^{2+}$ across membranes, but the members of the CorA family can also transport other divalent cation such as cobalt and nickel (Niegowski and Eshaghi, 2007). Besides, the CorA family includes the MRS2 protein from yeast that is thought to be an RNA splicing protein (Wiesenberger et al., 1992).

Sequences of mimpvic33 in the parental strains YAASMYPL61 was defined as A allele mimpvic33A, whereas $\mathrm{B}$ allele mimpvic33B was found in the strain YAASMYPL6-3. The lengths of the sequences mimpvic33A and mimpvic33B were 4,078 and $4,117 \mathrm{bp}$, and both contained 2 introns, with encoded proteins of 1311 and 1324 amino acids (Table 4), respectively. Compared with mimpvic33B, mimpvic33A had 4 amino acids difference, and 4-aa and 9-aa were missed. There was a 98.7\% amino acid sequence similarity between the two alleles (Supplementary Figure 2). The BLASTx analysis revealed that the predicted protein showed $26.5 \%$ amino acid similarity to the hypothetical protein of white truffle Tuber borchii (PUU75087), and had no any conserved protein domain family been found.

\section{Allelic Polymorphisms of mimpvic32 and mimpvic33}

Primer pairs P32 and P33 were designed to amplify the genes mimpvic32 and mimpvic33 (Supplementary Table 1). To verify allelic polymorphisms in different VCG populations, except for 15 strains from Zhao0001 population, each 10 single-ascospore strains were randomly selected from VCGA1, VCGA2, VCGB1, VCGB2, and VCG6611 to amplify mimpvi32 and mimpvi33, and the DNA sequence data were analyzed.

Thirty strains from VCGA1, VCGA2, and VCG6611, as well as YAASMYPL6-1, harbored the genes minpvic32A and minpvic33A. YAASMYPL6-3 and 20 strains from VCGB1 and VCGB2 carried minpvic32B and minpvic33B. In 15 strains of wild population Zhao0001, no A but $\mathrm{C}$ allele of the minpvic33 gene was detected (Supplementary Figure 2), and they had either minpvi32A and minpvi33B, or minpvi32B and minpvi33C (Supplementary Table 8).

For VCGA and VCGB, the phenotypes with vegetative compatibility were consistent with allelic polymorphisms of minpvic32 and minpvic33, but not for VCG6611 and Zhao0001 populations. Ten strains from VCG6611, similar to the strains from VCGA, had minpvic32A and minpvic33A; however, there was a type I barrage between VCG6611 and VCGA. In the wild population Zhao0001, though Zhao0001-28 and Zhao000129 harboring the same minpvic32B and minpvic33C were compatible, the other strains, such as Zhao0001-6, Zhao0001-8, Zhao0001-15, Zhao0001-21, and so on, carrying the same alleles displayed VI. Therefore, we speculate that besides minpvic32 and minpvic33, there were other genes involved in the regulation of VI in M. importuna.

\section{DISCUSSION}

Vegetative incompatibility, a form of nonself allorecognition, is ubiquitous in filamentous fungi and may limit the spread of viruses (Zhang et al., 2014) or deleterious genetic elements and 
TABLE 4 | The target scaffolds based on SNP and InDel association analysis.

\begin{tabular}{|c|c|c|c|c|c|c|c|}
\hline \multicolumn{4}{|c|}{ Target scaffolds based on SNP association analysis } & \multicolumn{4}{|c|}{ Target scaffolds based on InDel association analysis } \\
\hline Scaffold & AIISNP & AssoSNP_ED & AssoSNP_SNP-index & Scaffold & AllnDel & AssolnDel_ED & AssolnDel_InDel-index \\
\hline Scaffold50* & 179 & 179 & 179 & Scaffold50 & 43 & 42 & 43 \\
\hline Scaffold751* & 44 & 44 & 44 & Scaffold751 & 10 & 10 & 10 \\
\hline Scaffold40 & 49 & 12 & 49 & Scaffold1303 & 3 & 3 & 3 \\
\hline Scaffold613 & 12 & 6 & 10 & & & & \\
\hline Scaffold1388 & 12 & 4 & 12 & & & & \\
\hline Scaffold1798 & 8 & 7 & 7 & & & & \\
\hline Scaffold1876 & 6 & 4 & 6 & & & & \\
\hline Scaffold2066 & 4 & 3 & 4 & & & & \\
\hline
\end{tabular}

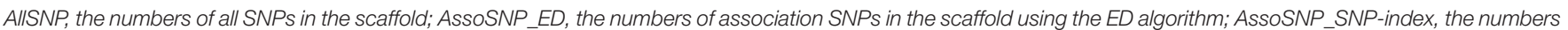

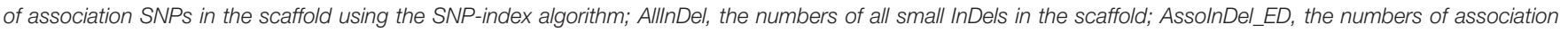
InDels in the scaffold using the ED algorithm; AssolnDel_InDel-index, the numbers of association InDels in the scaffold using the InDel-index algorithm.

*The final candidate scaffolds.
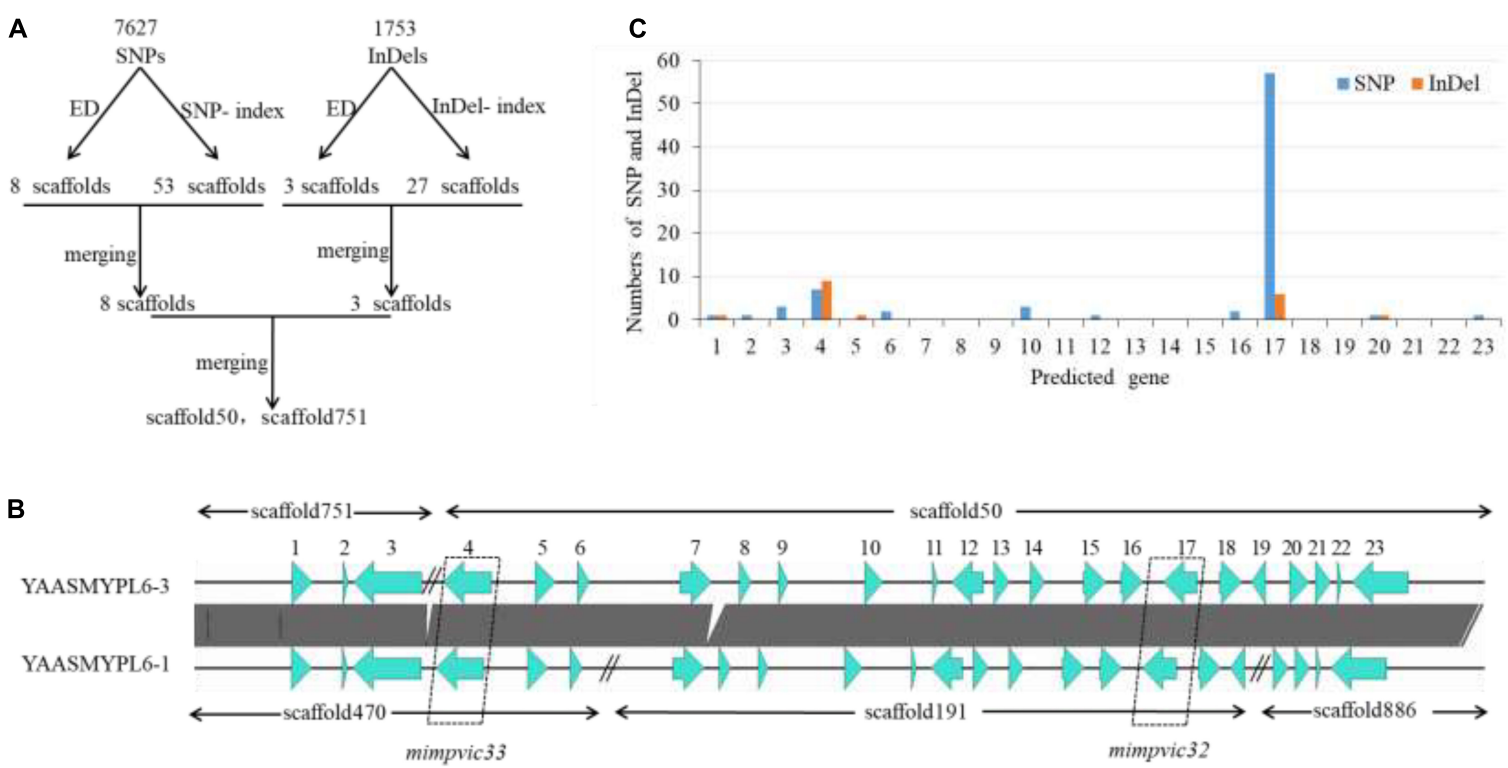

FIGURE 3 | Location of VI-related genes by Bulk Segregant Analysis. (A) Overview of the analysis workflow for screening the candidate scaffolds. (B) The comparative analysis of the YAASMYPL6-1 and YAASMYPL6-3 genomes showed that the scaffolds 50 and 751 were closely connected. (C) Comparison of the SNPS and InDels distributed in 23 predicted genes.

TABLE 5 | Allelic variation of the VI-related candidate genes mimpvic32 and mimpvic33.

\begin{tabular}{|c|c|c|c|c|c|c|c|c|c|c|}
\hline \multirow[t]{2}{*}{ Strain } & \multicolumn{5}{|c|}{ mimpvi32 } & \multicolumn{5}{|c|}{ mimpvi33 } \\
\hline & Allele type & $\begin{array}{c}\text { Intron } \\
\text { number }\end{array}$ & DNA (bp) & $\begin{array}{l}\text { Amino acids } \\
\text { (aa) }\end{array}$ & $\begin{array}{c}\text { GenBank } \\
\text { accession } \\
\text { number }\end{array}$ & Allele type & $\begin{array}{l}\text { Intron } \\
\text { number }\end{array}$ & DNA (bp) & $\begin{array}{c}\text { Amino } \\
\text { acids (aa) }\end{array}$ & $\begin{array}{c}\text { GenBank } \\
\text { accession } \\
\text { number }\end{array}$ \\
\hline YAASMYPL6-1 & A & 8 & 2,906 & 773 & OL303981 & A & 2 & 4,078 & 1,311 & OL303983 \\
\hline YAASMYPL6-3 & $\mathrm{B}$ & 8 & 2,875 & 773 & OL303980 & $\mathrm{B}$ & 2 & 4,117 & 1,324 & OL303982 \\
\hline Zhao0001-28 & $\mathrm{B}$ & 8 & 2,875 & 773 & OL303980 & C & 2 & 4,090 & 1,315 & OL303984 \\
\hline
\end{tabular}

prevent the plundering of resources (Pál1 et al., 2007). VI has been widespread in Morchella Mes-19, Morchella sextelata, and M. importuna (Liu et al., 2021). According to Yu and Zhao (2020), pairing the strains of $M$. importuna with $100 \%$ genetic similarity based on the ISSR (inter-simple sequence repeats) analysis resulted in the occurrence of antagonistic actions. Ma et al. (2017) isolated 15 strains from the cultivation regions of $M$. importuna in Nanyang city of China, and the results of 
confrontation tests showed that 101 out of 105 combinations were antagonistic. Our data also indicated that VI was a universal phenomenon among the strains of $M$. importuna, and the strains from different populations presented VI.

All 26 single-ascospore strains from the cultured ascocarps 6611-5 and 6611-7 were compatible and they formed a VCG with the parent strain 6611. We deduced that the two nuclei with the opposite mating types in strain 6611 were vegetatively compatible, thus indicating that all progenies from strain 6611 would be assigned to a VCG. However, the offspring from vegetatively incompatible parents was more complicated. The single-ascospore strains from incompatible parents were divided into four VCGs, and different manifestation of incompatibility exhibited between the inter-group strains. The VI interactions of VCGA1 and VCGB1 were consistent with those of the parental strains YAASMYPL6-1 and YAASMYPL63 , respectively, whereas the VCGA2 and VCGB2 presented new phenotypic characteristics and showed type I or type II barrages with the parents. In Morchella esculenta, no demarcation formed when monoascospore cultures from single ascus were paired, but horizontal or longitudinal fluffy zone occurred when confrontation tests were preformed between eight ascospore isolates of two asci of same ascocarp (Singh et al., 2006). The horizontal and longitudinal fluffy zone were similar to the type II barrages in this study. We speculated that the strains in VCGA1, VCGA2, VCGB1, and VCGB2 originated from different asci respectively, and among which distinct genetic recombination events occurred.

Our analyses indicated that in the genome of $M$. importuna, homologous sequences were detected only for het-c, un-24, het-c2, and two HET domain-containing genes, but they did not exhibit allelic polymorphisms between different VCGs. Moreover, all of them were not located in the candidate scaffolds obtained by the BSA. We speculate that these het genes may not control the VI reaction in $M$. importuna. The results of BSA allowed us to screen out the two VI-related mimpvic32 and mimpvic33 loci, with two and three alleles, respectively. However, with BSA results, we could only look for VI-related alleles, while the genes mediating nonallelic interactions could not be detected.

The members from VCGA1, VCGA2, and VCG6611, as well as YAASMYPL6-1, all carried mimpvic32A and mimpvic33A, but between the pairs, different barrage types were described. The strains of the zhao0001 population carried the same alleles of the mimpvic32 and mimpvic33 loci and also showed VI. These phenomena indicated that the VI control system in M. importuna was complicated, and there were more other allelic or nonallelic VI-related genes.

In $N$. crassa, mating-type locus acts as a vegetativeincompatibility locus, and the vegetative coexpression of opposite mating types is lethal (Beadle and Coonradt, 1944;

\section{REFERENCES}

Altschul, S. F., Madden, T. L., Schaffer, A. A., Zhang, J., Zhang, Z., Miller, W., et al. (1997). Gapped BLAST and PSI-BLAST: a new generation of protein database search programs. Nucleic Acids Res. 25, 3389-3402. doi: 10.1093/nar/25.17.3389
Garnjobst, 1953). However, mating type-associated VI was not found in most filamentous ascomycetes (Saupe, 2000). In this study, the strains from the same VCG could be MAT1-1 or MAT 1-2, and pairing the strains of the opposite mating type, with both vegetatively compatible and incompatible reactions, would occur. Therefore, in M. importuna, mating-type locus does not participate in the regulation of VI. But during the sexual phase, the mechanisms that regulate the ability of two vegetatively incompatible nuclei to coexist in the cytoplasm and undergo karyogamy are not yet clear.

\section{CONCLUSION}

The study indicated that VI was widespread in M. importuna and two types of barrages formed in the antagonistic reaction. Self/nonself-recognition in this fungus might not be regulated by the reported het genes, whereas, two genes mimpvic32 and mimpvic33 were associated with the VI reactions.

\section{DATA AVAILABILITY STATEMENT}

The datasets presented in this study can be found in online repositories. Raw sequences are deposited in the Sequence Read Archive under Bioproject PRJNA797544. The names of the repository/repositories and accession number(s) can be found in the article/Supplementary Material.

\section{AUTHOR CONTRIBUTIONS}

PL, YM, WC, and NT designed and performed the experiments, and analyzed the data. YZ conceived and designed the experiments. $\mathrm{HC}$ conceived and designed the experiments, and wrote the original manuscript. All authors contributed to the article and approved the submitted version.

\section{FUNDING}

This work was financially supported by National Natural Science Foundation of China (31960621) and China Agricultural Research System of MOF and MARA (CARS-20).

\section{SUPPLEMENTARY MATERIAL}

The Supplementary Material for this article can be found online at: https://www.frontiersin.org/articles/10.3389/fmicb. 2022.828514/full\#supplementary-material

Ashburner, M., Ball, C. A., Blake, J. A., Botstein, D., Butler, H., Cherry, J. M., et al. (2010). Gene ontology: tool for the unification of biology. the gene ontology consortium. Nat. Genet. 25, 25-29. doi: 10.1038/75556

Beadle, G. W., and Coonradt, V. L. (1944). Heterokaryosis in Neurospora crassa. Genetics 29, 291-308. 
Chai, H. M., Chen, L. J., Chen, W. M., Zhao, Q., Zhang, X. L., Su, K. M., et al. (2017). Characterization of mating-type idiomorphs suggests that Morchella importuna, Mel-20 and M. sextelata are heterothallic. Mycol. Prog. 16, 743-752. doi: 10.1007/s11557-017-1309-x

Chen, Q., and Zhang, J. X. (2010). An improved method to characterize fungal vegetative compatibility group(in Chinese). J. Microbiol. 30, 45-47.

Cingolani, P., Platts, A., Wang, L. L., Coon, M., Nguyen, T., Wang, L., et al. (2012). A program for annotating and predicting the effects of single nucleotide polymorphisms, SnpEff: SNPs in the genome of Drosophila melanogaster strain w1118; iso-2; iso-3. Fly 6, 80-92. doi: 10.4161/fly.19695

Cristina, O. M., and Myron, L. S. (2003). On the independence of barrage formation and heterokaryon incompatibility in Neurospora crassa. Fungal Genet. Biol. 38, 209-219. doi: 10.1016/S1087-1845(02)00533-9

Daskalov, A., Heller, J., Herzog, S., Fleibner, A., and Glass, N. L. (2017). Molecular mechanisms regulating cell fusion and heterokaryon formation in filamentous fungi. Microbiol. Spectr. 5, FUNK-0015-2016. doi: 10.1128/microbiolspec. FUNK-0015-2016

Debuchy, R., and Turgeon, B. G. (2006). "Mating-type structure, evolution, and function in euascomycetes," in Growth, Differentiation and Sexuality, eds K. Esser, R. Fischer, and U. Kües (Berlin: Springer-Verlag), 293-323. doi: 10.1007/ 3-540-28135-5_15

Deng, Y. Y., Li, J. Q., Wu, S. F., Zhu, Y. P., Cai, Y. W., and He, F. C. (2006). Integrated $\mathrm{nr}$ database in protein annotation system and its localization. Comput. Eng. 32, 71-74.

Espagne, E., Balhadère, P., Penin, M. L., Barreau, C., and Turcq, B. (2002). HET-E and HET-D belong to a new subfamily of WD40 proteins involved in vegetative incompatibility specificity in the fungus Podospora anserina. Genetics 161, 71-81. doi: 10.1093/genetics/161.1.71

Esser, K. (2016). "Heterogenic incompatibility in fungi," in The Mycota. I. Growth, Differentiation And Sexuality, 3rd Edn, ed. J. Wendlan (Cham: Springer), 103-131. doi: 10.1007/978-3-319-25844-7_6

Garnjobst, L. (1953). Genetic control of heterokaryosis in neurospora crassa. Am. J. Bot. 40, 607-614. doi: 10.2307/2438448

Glass, N. L., and Kuldau, G. A. (1992). Mating type and vegetative incompatibility in filamentous ascomycetes. Annu. Rev. phytopathol. 30, 201-224. doi: 10.1146/ annurev.py.30.090192.001221

Iotti, M., Rubini, A., Tisserant, E., Kholer, A., Paolocci, F., and Zambonelli, A. (2012). Self/nonself recognition in Tuber melanosporum is not mediated by a heterokaryon incompatibility system. Fungal Biol. 116, 261-275. doi: 10.1016/j. funbio.2011.11.009

Jeannmougin, F., Thompson, J. D., Gouy, M., Higgins, D. G., and Gibson, T. J. (1998). Multiple sequence alignment with Clustal X. Trends Biochem. Sci. 23, 403-405. doi: 10.1016/S0968-0004(98)01285-7

Kehres, D. J., Lawyer, C. H., and Maguire, M. E. (1998). The CorA magnesium transporter gene family. Microb. Comp. Genomics 3, 151-169. doi: 10.1089/omi. 1.1998.3.151

Li, H., and Durbin, R. (2009). Fast and accurate short read alignment with burrowswheeler transform. Bioinformatics 25, 1754-1760. doi: 10.1093/bioinformatics/ btp324

Liu, P., Ma, Y. H., Zhao, Y. C., Chai, H. M., and Li, Y. (2021). Sexual and vegetative compatibility of single ascospore isolation in the genus Morchella. J. Edible Fungi 28, 40-47. doi: 10.16488/j.cnki.1005-9873.2021 .01 .006

Liu, Q. Z., Ma, H. S., Zhang, Y., and Dong, C. H. (2017). Artificial cultivation of true morels: current state, issues and perspectives. Crit. Rev. Biotechnol. 38, 1-13. doi: 10.1080/07388551.2017.1333082

Ma, Y., Shen, J. D., Song, J. F., Ma, L. J., Lu, C. Y., Wang, S. C., et al. (2017). Antagonistic tests between different cultivate strains of Morel. Edible Fungi China 36, 80-82. doi: 10.13629/j.cnki.53-1054.2017 .05 .020

McKenna, A., Hanna, M., Banks, E., Sivachenko, A., Cibulskis, K., Kernytsky, A., et al. (2010). The Genome analysis toolkit: a mapreduce framework for analyzing next generation DNA sequencing data. Genome Res. 20, 1297-1303. doi: $10.1101 /$ gr.107524.110

Micali, C. O., and Smith, M. L. (2003). On the independence of barrage formation and heterokaryon incompatibility in Neurospora crassa. Fungal Genet. Biol. 38, 209-219.

Mirco, I., Andrea, R., Emilie, T., Annegret, K., Francesco, P., and Alessandra, Z. (2012). Self/nonself recognition in Tuber melanosporum is not mediated by a heterokaryon incompatibility system. Fungal Biol. 116, 261-275.

Niegowski, D., and Eshaghi, S. (2007). The CorA family: structure and function revisited. Cell. Mol. Life Sci. 64, 2564-2574. doi: 10.1007/s00018-007 -7174-Z

Pál1, K., van Diepeningen, A. D., Varga, J., Hoekstra, R. F., Dyer, P. S., and Debets, A. J. M. (2007). Sexual and vegetative compatibility genes in the aspergilli. Stud. Mycol. 59, 19-30. doi: 10.3114/sim.2007.59.03

Paoletti, M. (2016). Vegetative incompatibility in fungi: from recognition to cell death, whatever does the trick. Fungal Biol. Rev. 30, 152-162. doi: 10.1016/j.fbr. 2016.08.002

Saupe, S. J. (2000). Molecular genetics of heterokaryon incompatibility in filamentous ascomycetes. Microbiol. Mol. Biol. Rev. 64, 489-502. doi: 10.1128/ MMBR.64.3.489-502.2000

Saupe, S. J., Clavé, C., and Bégueret, J. (2001). Vegetative incompatibility in filamentous fungi: podospora and neurospora provide some clues. Curr. Opin. Microbiol. 3, 608-612. doi: 10.1016/S1369-5274(00 )00148-X

Singh, S. K., Tiwari, M., Kamal, S., Yadav, M. C., and Singh, S. (2006). Cytology of hyphal interactions in ascocarp cultures of Morchella esculenta. Mushroom Res. $15,11-17$.

Turgeon, B. G., and Yoder, O. C. (2000). Proposed nomenclature for mating type genes of filamentous ascomycetes. Fungal Genet. Biol. 31, 1-5. doi: 10.1006/fgbi. 2000.1227

Wiesenberger, G., Waldherr, M., and Schweyen, R. J. (1992). The nuclear gene MRS2 is essential for the excision of group II introns from yeast mitochondrial transcripts in vivo. J. Biol. Chem. 267, 6963-6969. doi: 10.1016/0014-5793(92) 80867-G

Yu, F. M., and Zhao, Q. (2020). Genetic diversity of hyphae sprouted from different single spores and different germ pores of morels. Mycosystema 39, 1117-1129. doi: 10.13346/j.mycosystema.200075

Zhang, D. X., Spiering, M. J., Dawe, A. L., and Nuss, D. L. (2014). Vegetative incompatibility loci with dedicated roles in allorecognition restrict mycovirus transmission in chestnut blight fungus. Genetics 197, 701-714. doi: 10.1534/ genetics.114.164574

Zhao, J. H., Gladieux, P., Hutchison, E., Bueche, J., Hall, C., Perraudeau, F., et al. (2015). Identification of alorecognition loci in Neurospora crassa by genomics and evolutionary approaches. Mol. Biol. Evol. 32, 2417-2432. doi: 10.1093/ molbev/msv125

Conflict of Interest: The authors declare that the research was conducted in the absence of any commercial or financial relationships that could be construed as a potential conflict of interest.

Publisher's Note: All claims expressed in this article are solely those of the authors and do not necessarily represent those of their affiliated organizations, or those of the publisher, the editors and the reviewers. Any product that may be evaluated in this article, or claim that may be made by its manufacturer, is not guaranteed or endorsed by the publisher.

Copyright (c) 2022 Chai, Liu, Ma, Chen, Tao and Zhao. This is an open-access article distributed under the terms of the Creative Commons Attribution License (CC BY). The use, distribution or reproduction in other forums is permitted, provided the original author(s) and the copyright owner(s) are credited and that the original publication in this journal is cited, in accordance with accepted academic practice. No use, distribution or reproduction is permitted which does not comply with these terms. 